\title{
Investigation on the existence of a 'Hillert regime' in normal grain growth
}

\author{
Vishal Yadav, Nele Moelans \\ Dept. of Materials Engineering, KU Leuven, Kasteelpark Arenberg 44 Bus 2450, Leuven 3001, Belgium
}

\begin{abstract}
The existence of a 'Hillert regime' in 3D normal grain growth, where the grain size distributions (GSDs) at different time-steps match the Hillert distribution during parabolic grain growth, is investigated for different initial GSDs using large-scale phase-field simulations. The short-lived 'Hillert regime' was present in the early-stage only in few cases. The GSDs obtained at a later-stage for all cases are: self-similar over long period; independent of the initial GSDs; and wider than the Hillert distribution. Also, the topological properties in the 'Hillert regime' were different from that in the later-stage self-similar regime.
\end{abstract}

Keywords: Grain growth, Hillert distribution, Simulation and modeling

The commonly accepted characteristics of steady-state normal grain growth (NGG) in an isotropic single-phase system [1-3] after a transient regime are: parabolic growth rate and unique self-similar grain size distribution (GSD) and grain topology distribution (GTD) (also known as distribution of number of faces). In 1965, Hillert [4] proposed an analytical expression for the steady-state GSD based on a mean-field treatment of the evolution of the individual grains. Battaile and Holm [5] observed, based on 2D Monte Carlo Potts simulations, that the self-similar steady-state GSD deviates from the Hillert distribution. Recent large scale 3D simulations [6] 8] using the multi-phase field model [9] have shown inconsistent results during NGG. In a first study, Kim et al. [6] found that the steady-state GSDs at different time-steps were independent of the initial GSDs and in excellent agreement with the Hillert distribution. They concluded

\footnotetext{
* Corresponding authors

Email addresses: vishal yadav@student.kuleuven.be (Vishal Yadav), Nele.Moelans@kuleuven. be (Nele Moelans)
} 
that the Hillert theory can be regarded as an accurate description of 3D steady-state NGG. In a second study, Suwa et al. [7] noted that the steady-state GSDs were wider than the Hillert distribution. In a third study, Kamachali and Steinbach [8] observed 'Hillert regime' during parabolic grain growth, where the GSDs in the early-stage match the Hillert distribution for some time-steps. Later, as simulation progressed, the GSDs evolved towards a wider distribution, which were considered as the self-similar GSDs. They also claimed that 'the same behaviour has been observed from simulation boxes of different sizes and initial distributions', but, unlike Kim et al. [6], no supporting material was given. Furthermore, Kamachali and Steinbach [8] observed that the GTDs, unlike the GSDs, were invariant throughout the Hillert and later-stage regimes. Other large-scale computer simulations using various numerical schemes [10 13] did not observe a 'Hillert regime' during NGG. The mean number of faces in Kim et al. [6] study was $\langle F\rangle=13.4$ whereas others have reported $\langle F\rangle \approx 13.7$ [7, 10 13]. Kamachali and Steinbach [8] did not report on the $\langle F\rangle$ value. The goal of this paper is to understand the discrepancies between previously published different 3D multi-phase field simulation results and to study the effect of the initial grain size distribution on the existence of a Hillert regime in NGG.

In the present study, simulations are carried out using a continuum phase-field model [14]. The details of the model [15], numerical scheme [16], model parameters (like, $\Delta t$ time-step, etc), initial GSDs, calculation of grain radius $R$ and mean grain size $\langle R\rangle$ are given in the 'Supplementary material'. The system size and other simulation parameters in this paper are identical to the Kamachali and Steinbach [8] study. Six different initial grain size distributions are considered here. The initial shape of the GSDs after $100 \Delta t$ time-step (where $\Delta t=0.1 \mathrm{sec}$ ) is shown in Fig. 1. For Cases 1-4 and 6, 30,000 grains $(N)$ were present at the start of the simulation. For Case 5, the initial grain structure contained 50,000 grains. In all cases, grains were initialized as spherical grains and distributed randomly over the $3 \mathrm{D}$ system. The initial grain radii were selected to obtain the desired initial GSDs (further details in 'Supplementary material'). For Case 3, the shape of the GSD is similar to the Hillert distribution at 100 $\Delta t$ (Fig. 1). For Cases 4-6, the tail of the initial GSD is longer than the Hillert distribution. 
The evolution of the square of the $\langle R\rangle$ with time for all cases is shown in Fig. 2a. The inset figure in Fig. 2a shows the evolution of the $\langle R\rangle$ from $100 \Delta t-4000 \Delta t$. The evolution of the GSDs for Cases 1-5 are shown in Figs. 3a-3c, 3g and 3h. We distinguished two regimes during the parabolic grain growth: 1) Regime I, part of an early-stage regime $(100 \Delta t-4000 \Delta t)$, where quasi-steady-state (QSS) is observed. In the QSS, GSDs are constant for some time before they change shape. For all cases, the duration of this regime was different; and 2) Regime II, the later-stage regime $(4000 \Delta t-20000 \Delta t)$, where the GSDs remained invariant (self-similar) over this period, and are the same for Cases 1-5. In the same way, the evolution of the GTDs for Cases 1-5 is presented in Figs. 3d-3f, 3j and 3k. The standard deviation $(\sigma)$ of the normalized grain sizes $(R /\langle R\rangle)$, mean number of faces $\langle F\rangle$ and second moment $(\mu)$ of number of faces $F$ of all grains are shown in Figs. 2b-2d for all cases. Further details are presented in Table 1. For Case 6, the steady-state was achieved later than in Cases 1-5. Finally, the GSD and GTD, obtained for Case 1, are compared with previously published results in Fig. 4.

The linear relation between the square of the $\langle R\rangle$ and time $(t)$ in Regime I and Regime II for all cases (see Fig. 2a) indicates that parabolic growth kinetics, $\langle R\rangle^{2}-\left\langle R_{o}\right\rangle^{2}=K t$, are followed for both regimes, where $\left\langle R_{o}\right\rangle$ is the mean grain radius at the start of parabolic growth and $K$ is a kinetic coefficient. The GSDs for Regime I (see Fig. 3) suggest that QSS GSDs are observed for Cases 1-5, but the Hillert Regime is observed only for Cases 1-3. The $K_{I}, \sigma_{I},\left\langle F_{I}\right\rangle$ and $\mu_{I}$ in this Hillert Regime have similar values for Cases 1-3 (see Table 1a). However, the GTDs in the Hillert regime behaved differently in the different Cases. For Cases 1-2, the GTDs are constant in Regime I, while this is not the case for Case 3. For Cases 4-5, where the QSS GSDs do not match the Hillert distribution, the $K_{I}$ and $\sigma_{I}$ are different, whereas the $\left\langle F_{I}\right\rangle$ and $\mu_{I}$ are similar to those in the Hillert regime in Cases 1-3. For Case 4, the GTD in Regime I is constant, while this is not the case for Case 5. Suwa et al. [7] reported that the $\langle F\rangle=14$ in the early-stage of their simulation, which is similar to the $\left\langle F_{I}\right\rangle$ value in the present study. However, Kim et al. [6] found that the $\langle F\rangle=13.4$ in the Hillert regime. Our results from Regime I for the different Cases show thus large variation in GSDs, GTDs, $K_{I}, \sigma_{I},\left\langle F_{I}\right\rangle$ and $\mu_{I}$ despite showing 
parabolic grain growth. This indicates that the Hillert regime cannot be considered as a unique regime in the early-stage of parabolic grain growth.

On the other hand, the GSDs, GTDs, $K_{I I}, \sigma_{I I},\left\langle F_{I I}\right\rangle$ and $\mu_{I I}$ of Regime II for all cases are similar and invariant despite having different initial GSDs (see Table $1 \mathrm{~b}$ ). The $K_{I I}$ obtained in this study is also close to the kinetic coefficient $K=5.1 \times 10^{-15} \mathrm{~m}^{2} / \mathrm{s}$ obtained in [8]. The $\left\langle F_{I I}\right\rangle$ for all cases also agrees with the $\langle F\rangle$ value published in previous results [7, 10 13]. The averaged GSDs and GTDs over Regime II are similar to the steady-state GSD and GTD reported by Elsey et al. [10] (see Figs. 3a-3h, 3j and 3k). In Figs. 3i and 31 , the GSDs and GTDs for Regime II are compared for Cases 1-6 and with the results of Kamachali and Steinbach [8]. The GSD from [8] at 8200 $\Delta t$ looks similar to our GSDs, however, the peak is shifted towards lower grain sizes and a few large grain sizes are also present in their simulations (see Fig. 3i). The peak position of the GTD from [8] at $1800 \Delta t$ matches with the present results, but the match at the 'ear' and 'tail' of the distributions is poor (see Fig. 31). The GTD from the later-stage (9400 $\Delta t$ [8]) matched the peak position and tail end but the ear position showed the existence of more grains belonging to $F \leq 6$ topological class than the present study. Different from our findings, Kamachali and Steinbach [8] observed that the GTDs remained invariant over Regime I and Regime II. They suggested that the possible reason behind invariant GTD and different GSD shapes during parabolic grain growth may be due to a non-linear relation between the $\sqrt{F}$ and grain sizes $R$. However, in our study, the GTDs from Regime I and Regime II are different for different initial GSDs, showing that their observation of an invariant GTD over the two regimes is not generally true. In Fig. 4, the GSD and GTD for Regime II from our simulations agree well with the previous simulation results [7, 12, 13, 17] corresponding to the steady-state regime. The Rios distribution [18] (with $\nu=3.2$ ) also matches our GSD results, whereas, the log-normal distribution fit [19] (with $\mu=0.0529$ and $\sigma=0.4125)$ showed a poor match. Krill and Chen [20] observed parabolic grain growth with $\langle F\rangle=13.7$. However, their GSD and GTD show a poor match with our results. It was, however, already suggested [6] that the simulations in the Krill and Chen [20] study may not represent a steady-state NGG. The GTD shape in the Kim 
et al. [6] study is similar to our steady-state GTD but their GSD is similar to the Hillert distribution. The simulations in this study are carried out with the continuum phase-filed model and Moelans et al. 221] confirmed that the continuum phase-field model and the multi-phase field model should give similar grain growth results. However, unlike the findings of Kamachali and Steinbach [8], we find that the topological properties in the Hillert regime are different from those in the later-stage self-similar regime and that the Hillert regime is dependent on the initial GSD.

It is important to note that the analytical solutions for parabolic grain growth and the Hillert distribution do not contain topological information, while microstructure evolution is also constrained by the presence of grain topology. Our results show the importance of verifying not only the self-similar GSDs but also the invariant topological properties during parabolic grain growth when determining the steady-state regime.

In summary, based on large-scale grain growth simulations starting from six different initial GSDs, we conclude that only the later-stage microstructure evolution (Regime II) is a self-similar regime and independent of the initial GSD. Thus, the Hillert regime, whose existence is dependent on the initial GSD during parabolic grain growth, does not represent a steady-state regime in normal grain growth.

\section{Acknowledgements}

This work was supported by the Research Foundation Flanders (FWO-Vlaanderen, Belgium, G.0362.09). N.M. has received funding from the European Research Council (ERC) under the European Union's Horizon 2020 research and innovation program (grant agreement $n^{o}$ 714754). V.Y. would like to thank Amy Van den Bulck for discussion.

\section{Appendix A. Supplementary material}

\section{References}

[1] W. W. Mullins, J. Appl. Phys. 59 (1986) 1341. URL: http://scitation.aip.org/ content/aip/journal/jap/59/4/10.1063/1.336528, doi:10.1063/1.336528. 
[2] H. V. Atkinson, Acta Met. 36 (1988) 469-491. URL: http://www.sciencedirect. com/science/article/pii/000161608890079X. doi:10.1016/0001-6160(88) 90079-X.

[3] A. Rollett, F. Humphreys, G. S. Rohrer, M. Hatherly, Recrystallization and Related Annealing Phenomena: Second Edition, 2004. doi:10.1016/B978-0-08-044164-1. X5000-2.

[4] M. Hillert, Acta Metall. 13 (1965) 227-238. URL: www.sciencedirect.com/ science/article/pii/0001616065902002, doi:10.1016/0001-6160(65)90200-2.

[5] C. C. Battaile, E. A. Holm, in: Weiland, H and Adams, BL and Rollett, AD (Ed.), Grain growth in polycrystalline materials III, TMS, 1998, p. 119.

[6] S. G. Kim, D. I. Kim, W. T. Kim, Y. B. Park, Phys. Rev. E. Stat. Nonlin. Soft Matter Phys. 74 (2006) 061605. URL: http://journals.aps.org/pre/abstract/ 10.1103/PhysRevE.74.061605, doi:10.1103/PhysRevE.74.061605.

[7] Y. Suwa, Y. Saito, H. Onodera, Materials Transactions 49 (2008) 704-709. doi:10. 2320/matertrans.MRA2007225.

[8] R. D. Kamachali, I. Steinbach, Acta Mater. 60 (2012) 2719-2728. URL: http://www. sciencedirect.com/science/article/pii/S1359645412000754 doi $10.1016 / \mathrm{j}$. actamat.2012.01.037.

[9] I. Steinbach, F. Pezzolla, Phys. D Nonlinear Phenom. 134 (1999) 385-393. URL: http://www.sciencedirect.com/science/article/pii/S0167278999001293. doi:10.1016/S0167-2789(99)00129-3.

[10] M. Elsey, S. Esedoglu, P. Smereka, Proc. R. Soc. A Math. Phys. Eng. Sci. 467 (2011) 381-401. URL: http://rspa.royalsocietypublishing.org/cgi/doi/10. 1098/rspa.2010.0194. doi:10.1098/rspa.2010.0194

[11] E. A. Lazar, J. K. Mason, R. D. MacPherson, D. J. Srolovitz, Acta Mater. 59 
(2011) 6837-6847. URL: http://www.sciencedirect.com/science/article/pii/ S1359645411005386, doi:10.1016/j . actamat.2011.07.052.

[12] J. K. Mason, E. A. Lazar, R. D. MacPherson, D. J. Srolovitz, Phys. Rev. E 92 (2015) 063308. URL: http://link.aps.org/doi/10.1103/PhysRevE.92.063308. doi:10.1103/PhysRevE.92.063308.

[13] D. Zöllner, P. Streitenberger, P. Rios, Comput. Mater. Sci. 113 (2016) 11-20. doi:10. $1016 /$ j.commatsci.2015.11.022.

[14] D. Fan, L.-Q. Chen, Acta Mater. 45 (1997) 611-622. URL: http: //wWW.sciencedirect.com/science/article/pii/S1359645496002005. doi: $10.1016 / \mathrm{S} 1359-6454(96) 00200-5$.

[15] N. Moelans, B. Blanpain, P. Wollants, Phys. Rev. B 78 (2008) 024113. URL: http: //link.aps.org/doi/10.1103/PhysRevB.78.024113. doi:10.1103/PhysRevB.78. 024113.

[16] L. Vanherpe, N. Moelans, B. Blanpain, S. Vandewalle, Phys. Rev. E 76 (2007) 056702. URL: http://link.aps.org/doi/10.1103/PhysRevE.76.056702, doi:10. 1103/PhysRevE. 76.056702.

[17] E. Miyoshi, T. Takaki, M. Ohno, Y. Shibuta, S. Sakane, T. Shimokawabe, T. Aoki, npj Comput. Mater. 3 (2017) 25. URL: http://dx.doi.org/10.1038/ s41524-017-0029-8, doi:10.1038/s41524-017-0029-8.

[18] P. Rios, T. Dalpian, V. Brandão, J. Castro, A. Oliveira, Scr. Mater. 54 (2006) 1633-1637. URL: http://www.sciencedirect.com/science/article/pii/ S135964620600056X. doi:10.1016/j.scriptamat.2006.01.007.

[19] M. Fátima Vaz, M. Fortes, Scr. Metall. 22 (1988) 35-40. URL: http: //www.sciencedirect.com/science/article/pii/S0036974888803028. doi:10. 1016/S0036-9748(88)80302-8. 
[20] C. Krill, L.-Q. Chen, Acta Mater. 50 (2002) 3059-3075. URL: http: //www.sciencedirect.com/science/article/pii/S1359645402000848. doi:10. 1016/S1359-6454(02) 00084-8.

[21] N. Moelans, F. Wendler, B. Nestler, Comput. Mater. Sci. 46 (2009) 479-490. doi:10. 1016/j.commatsci.2009.03.037. 


\section{List of Figures}

$1 \quad$ Initial grain size distributions (GSDs) for all 6 cases considered in this paper. The GSDs of all 6 cases are normalized with $R_{c r}$ where $R_{c r}=$ $9 / 8 \cdot\langle R\rangle$. The total number of grains present at $100 \Delta t$ are shown in the legend. The Hillert 3D distribution is added for comparison. . . . . . . . 10

2 Evolution of the square of the mean grain radius $\langle R\rangle$, standard deviations $\sigma$ of all normalized grain sizes, mean number of faces $\langle F\rangle$ and second moment $\mu$ of the number of faces with time for the 6 Cases. The inset figure in Fig. 2a shows the evolution of the mean grain radius in the earlystage regime I $(100 \Delta t-4000 \Delta t)$. The vertical broken line in these figures indicates start of the Regime II for Cases 1-5. . . . . . . . . . . . . . . . 11

3 Grain size distributions and grain topology distributions in Regime I and II for all cases. The GSDs and GTDs over Regime I are shown at individual time-steps in the Figs. 3a-3h, 3j and 3k, while they are averaged over the time steps $4000 \Delta t-20000 \Delta t$ for Regime II. In Figs. 3i and 31, the GSDs and GTDs from Regime II of all cases are compared. The GSDs and GTDs are also compared with previous results [8, [10].

$4 \quad$ Comparison of grain size and grain topology distribution of Regime II for Case 1 with those obtained in previous simulation studies [6, 17, 12, 13, 17, 20] are shown in Fig. 4 a and b respectively. The analytical distributions [18, 19] are also compared in Fig. 44a. The grain sizes are normalized with $\langle R\rangle$ instead of $R_{c r}$. . . . . . . . . . . . . . . . . . . . . 13

\section{List of Tables}

1 Comparison of growth kinetics $K\left(10^{-15} \mathrm{~m}^{2} / \mathrm{s}\right)$, standard deviation of normalized grain sizes $\sigma$, mean number of faces $\langle F\rangle$ and second moment $\mu$ of number of faces $F$ in Regime I and Regime II. . . . . . . . . . . . . . . . 10 


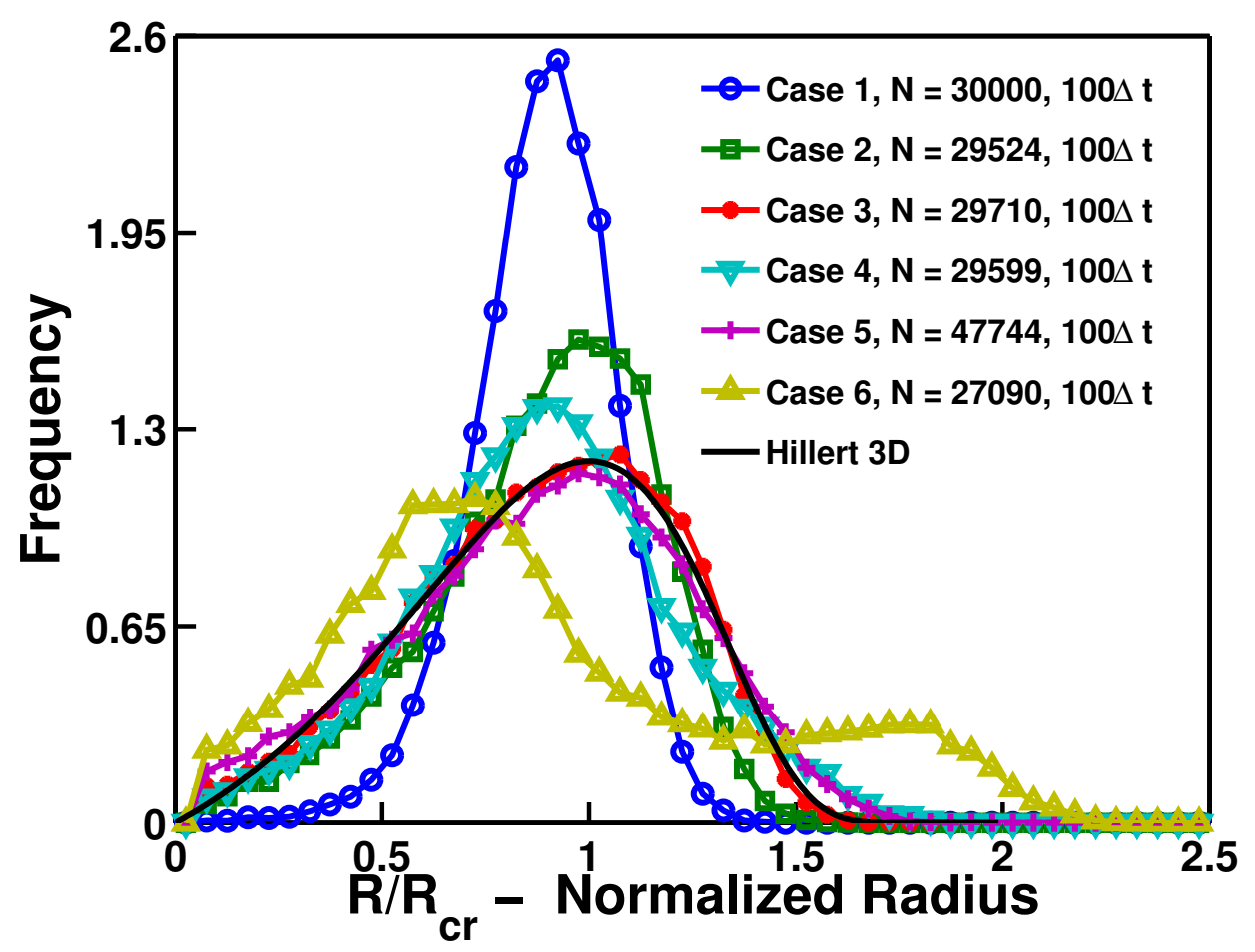

Fig. 1. Initial grain size distributions (GSDs) for all 6 cases considered in this paper. The GSDs of all 6 cases are normalized with $R_{c r}$ where $R_{c r}=9 / 8 \cdot\langle R\rangle$. The total number of grains present at $100 \Delta t$ are shown in the legend. The Hillert $3 \mathrm{D}$ distribution is added for comparison.

Table 1. Comparison of growth kinetics $K\left(10^{-15} \mathrm{~m}^{2} / \mathrm{s}\right)$, standard deviation of normalized grain sizes $\sigma$, mean number of faces $\langle F\rangle$ and second moment $\mu$ of number of faces $F$ in Regime I and Regime II.

(a) Regime I

\begin{tabular}{c|cccc}
\hline GSD & $K_{I}$ & $\sigma_{I}$ & $\left\langle F_{I}\right\rangle$ & $\mu_{I}$ \\
\hline Case 1 & 5.27 & $0.35 \pm 0.0030$ & $14.04 \pm 0.024$ & $23.29 \pm 0.31$ \\
Case 2 & 4.85 & $0.35 \pm 0.0045$ & $14.01 \pm 0.032$ & $23.34 \pm 0.54$ \\
Case 3 & 5.00 & $0.35 \pm 0.0053$ & $14.03 \pm 0.029$ & $22.89 \pm 1.54$ \\
Case 4 & 6.45 & $0.37 \pm 0.0024$ & $13.89 \pm 0.018$ & $27.02 \pm 0.41$ \\
Case 5 & 5.73 & $0.38 \pm 0.0072$ & $13.97 \pm 0.038$ & $26.65 \pm 1.11$ \\
Case 6 & 5.27 & $0.35 \pm 0.0058$ & $13.89 \pm 0.026$ & $20.19 \pm 0.45$ \\
\hline
\end{tabular}

(b) Regime II

\begin{tabular}{c|cccc}
\hline GSD & $K_{I I}$ & $\sigma_{I I}$ & $\left\langle F_{I I}\right\rangle$ & $\mu_{I I}$ \\
\hline Case 1 & 4.98 & $0.38 \pm 0.010$ & $13.78 \pm 0.077$ & $24.53 \pm 0.60$ \\
Case 2 & 5.06 & $0.38 \pm 0.009$ & $13.78 \pm 0.049$ & $24.76 \pm 0.70$ \\
Case 3 & 5.04 & $0.37 \pm 0.006$ & $13.79 \pm 0.054$ & $23.36 \pm 0.82$ \\
Case 4 & 4.98 & $0.38 \pm 0.007$ & $13.76 \pm 0.051$ & $24.58 \pm 0.91$ \\
Case 5 & 5.27 & $0.38 \pm 0.006$ & $13.77 \pm 0.042$ & $24.23 \pm 1.09$ \\
Case 6 & 5.06 & $0.37 \pm 0.007$ & $13.79 \pm 0.029$ & $23.21 \pm 0.71$ \\
\hline
\end{tabular}



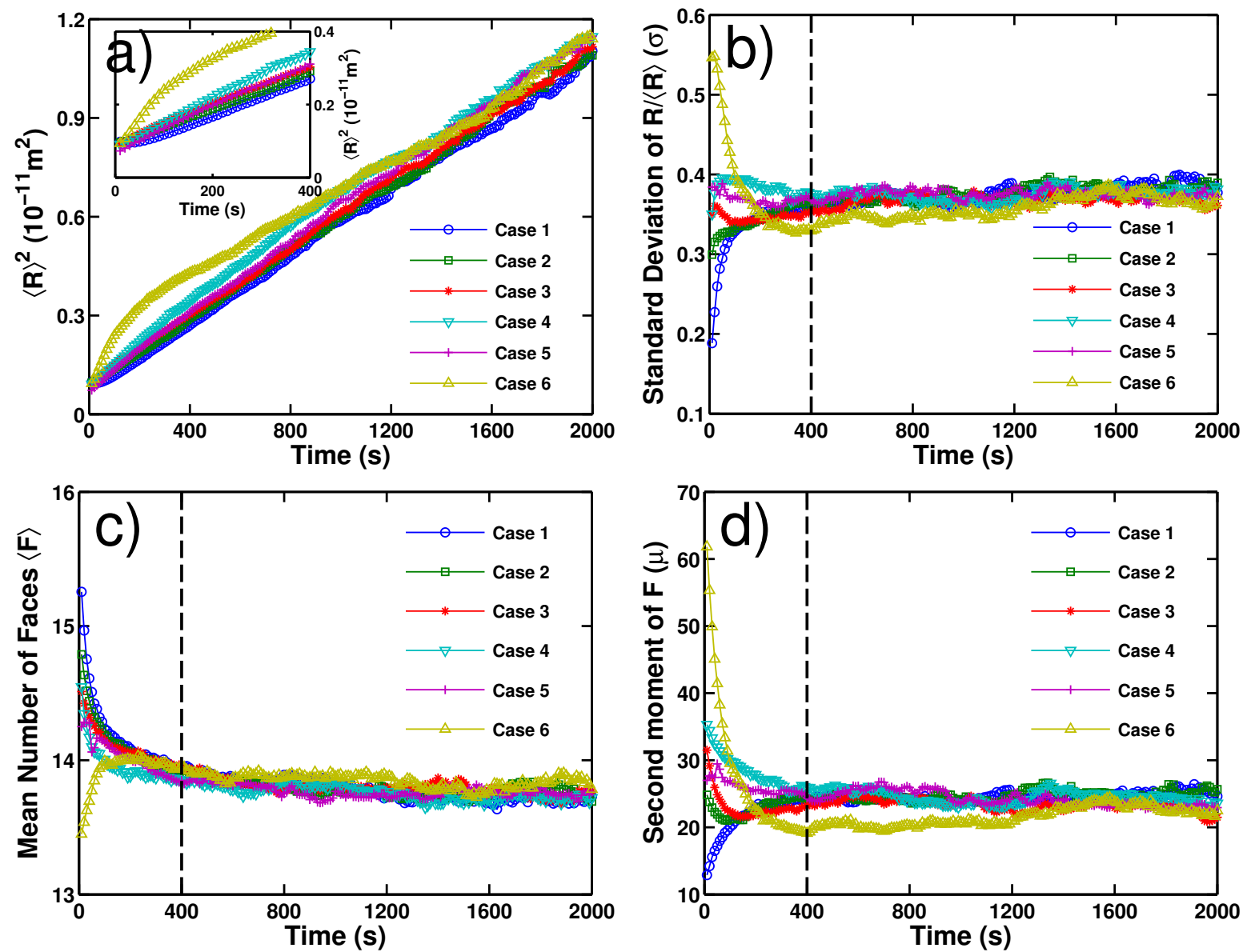

Fig. 2. Evolution of the square of the mean grain radius $\langle R\rangle$, standard deviations $\sigma$ of all normalized grain sizes, mean number of faces $\langle F\rangle$ and second moment $\mu$ of the number of faces with time for the 6 Cases. The inset figure in Fig. 2a shows the evolution of the mean grain radius in the early- stage regime I $(100 \Delta t-4000 \Delta t)$. The vertical broken line in these figures indicates start of the Regime II for Cases 1-5. 

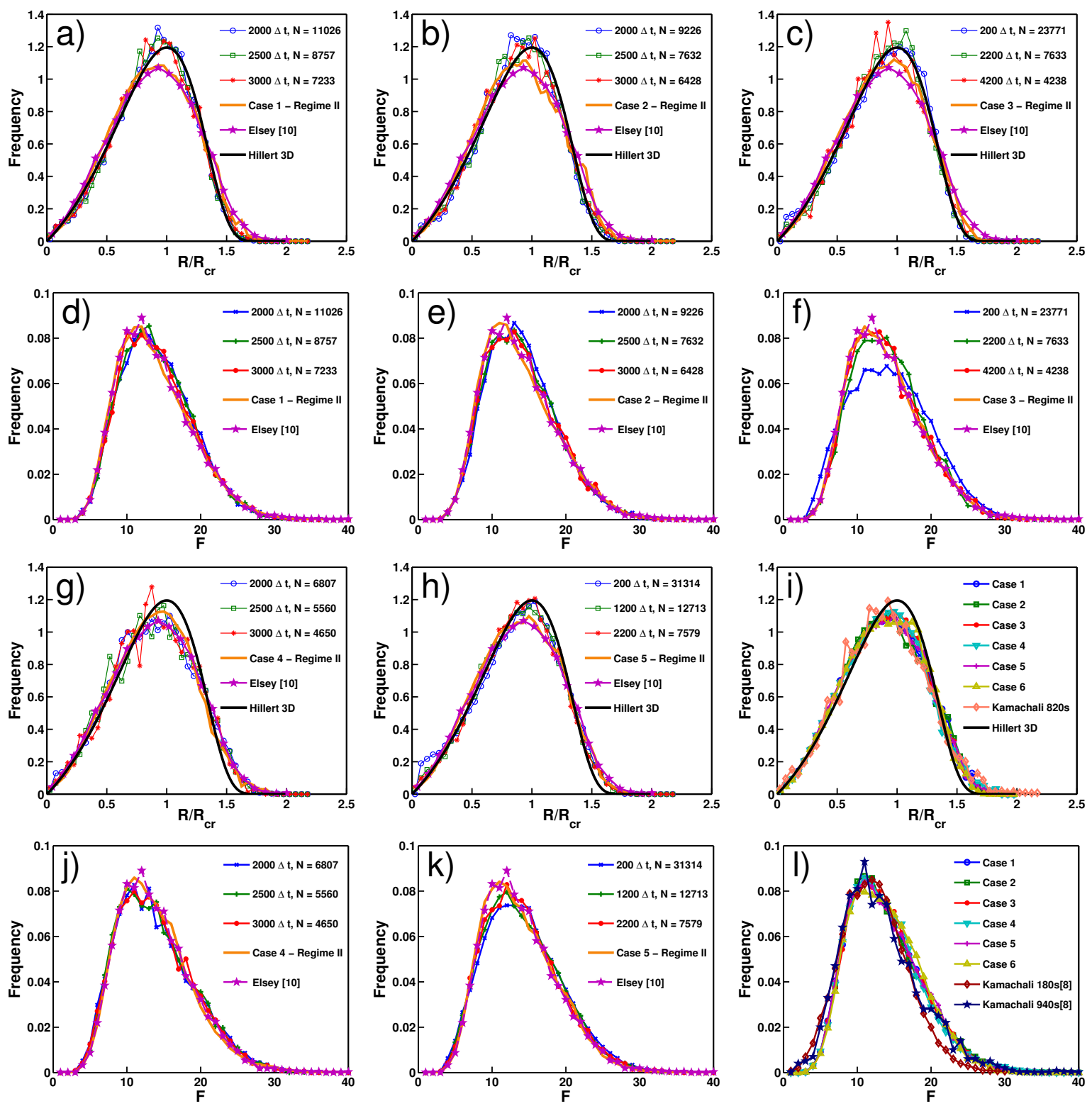

Fig. 3. Grain size distributions and grain topology distributions in Regime I and II for all cases. The GSDs and GTDs over Regime I are shown at individual time-steps in the Figs. 3a-3h, 3j and $3 \mathrm{k}$, while they are averaged over the time steps $4000 \Delta t-20000 \Delta t$ for Regime II. In Figs. $3 \mathrm{i}$ and 31, the GSDs and GTDs from Regime II of all cases are compared. The GSDs and GTDs are also compared with previous results [8, 10]. 

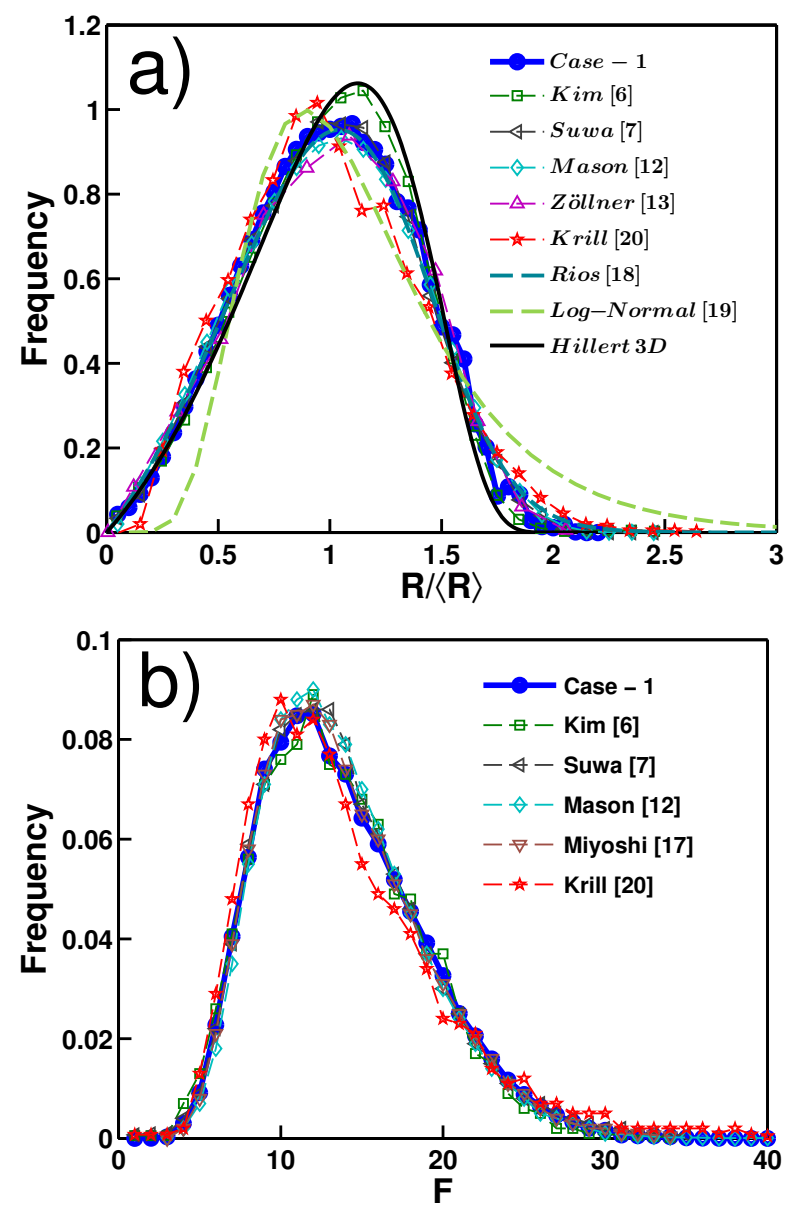

Fig. 4. Comparison of grain size and grain topology distribution of Regime II for Case 1 with those obtained in previous simulation studies [6, 7, 12, 13, 17, 20] are shown in Fig. 4a and b respectively. The analytical distributions [18, 19] are also compared in Fig. 4a. The grain sizes are normalized with $\langle R\rangle$ instead of $R_{c r}$. 